\title{
Fixed Point Acoustic Monitoring Investigation Report for Marine Biological Resources in an Open Water Channel of Taishan Nuclear Power Plant
}

\author{
XU Chuan ${ }^{1}$, HU Zhengchun ${ }^{1}$ \\ ${ }^{1}$ Taishan Nuclear Power Joint Venture Co., Ltd.
}

\begin{abstract}
To disclose changes of density of fish larva and other marine organisms in an open water channel before the open net interceptor, this acoustic monitoring point was determined in front of the first open net interceptor in the open seawater channel with considerations to monitoring functions of EK80 scientific echometer to various cold-source organism and the marine organism recovery efficiency of open net interceptor. The beam direction was perpendicular downward. Floating marine organism enters into the interceptor after passing through the acoustic beam, while the quantity of swimming fish larva into the interceptor is smaller under normal marine conditions since they have certain swimming ability.
\end{abstract}

\section{Introduction}

There's near shore aquiculture area in coastal sea areas surrounding the Taishan Nuclear Power Plant of China Guangdong Nuclear Power Company, which is characteristic of small water depth and large coverage of aquiculture facilities. The cold-source water point is set at the Dajin Island at $10 \mathrm{~km}$ out of the Taishan Nuclear Power Plant and an about $2.8 \mathrm{~km}$ long open water channel was built along the west side of Dajin Island. A good spawning site is formed as a result to the largescaled coverage of aquiculture facilities for oyster in surrounding sea areas. Marine organisms like fishes lay eggs here and there's a great quantity of larva ${ }^{1}$. Besides, the open water channel becomes an ideal place for activities of larva due to the good shelter effect from the wind. It is often to see a certain scale of larva cluster in the open water channel and many cuttlefish larva clusters can be observed at night under light ${ }^{2}$. Although there are many interceptors in the open water channel, including plane net and standing net (trash holding net bag), the quantity of marine organisms like small fishes (larva) increases in summer ${ }^{1}$, which threatens the safety operation of cold-sourced water uptaking ${ }^{6}$.

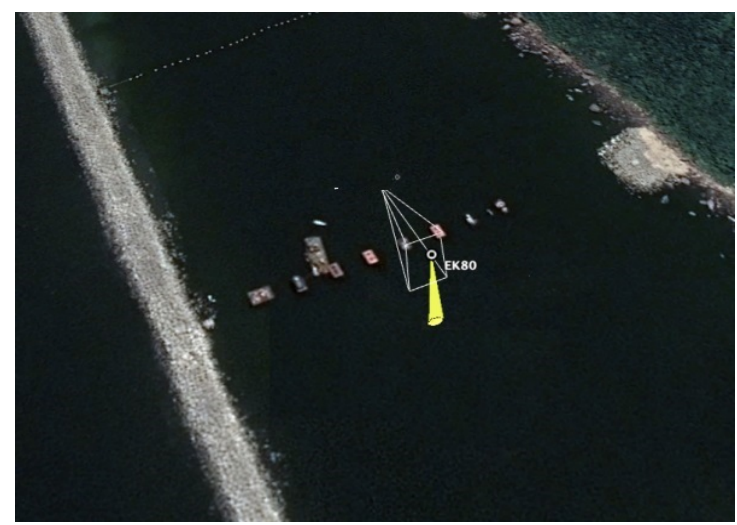

Fig.1 Fixed point acoustic monitoring scheme before the interceptor in an open water channel in the Taishan Nuclear Power Plant

\section{Fixed point monitoring method}

This fixed point monitoring began from 18:00 on May 20th, 2020 to 6:00 on May 21st, 2020. The monitoring point was set at the place $10 \mathrm{~m}$ in front of the $\# 5$ net (copper alloy net) in the first interceptor in the open water channel ${ }^{20}$. The broadband scientific echometer system (EK80, 90kHz-150kHz) of Simrad Company (Norway) was applied for acoustic measurement and echo data acquisition of organism like fishes in the fixed point ${ }^{34}$. GPS was not applied since it was a fixed point investigation without need of location data ${ }^{5}$. A DC12V storage battery was applied to output AC220V through the inverter to supply power to the scientific echometer and data acquisition $\mathrm{PC}^{14}$. The transducer was fixed at a side of the ship with a special support, with a submerged depth of $50 \mathrm{~cm}$. The investigation ship was a small diesel

\footnotetext{
* Corresponding author: xuchuan@ecgnpc.com.cn
} 
oiled tail on-hook fiberglass reinforced plastic structured boat without sunshade (about $6 \mathrm{~m}$ in length). The boat was fixed at $10 \mathrm{~m}$ in front of the interceptor.

\section{Results discussion}

\subsection{Echo mapping characteristics of marine organism}

The time compression mapping of monitored marine organism echoes from 18:00 on May 20th, 2020 to 6:00 on May 21st, 2020 is shown in Fig.2. The lower red strips are echoes from the seabed and the sine fluctuation of water depth is caused by tidal changes. The compressed red vertical stripped echoes are echoes of small fish clusters, while the scattered ones are echoes of single fish target. Since the echo intensity of single target is proportional to body length of fishes and the echo intensity of fish clusters is proportional to density, the larger red area with high echo intensity reflects the larger marine biomass that runs through the beam ${ }^{7}$. Therefore, it can be judged from Fig.2 that there's relatively high marine biomass from 18:00 to 24:00, which mainly distributed from $2 \mathrm{~m}$ to seabed. On contrary, there's relatively low marine biomass from 24:00 to 4:00, and the biomass increased again from 5:00 to 6:00.

The tide cycle was deduced according to changes of water depth. The marine organism density in the monitoring section was relatively high during ebbs, but it was relatively small during falls. In other words, based on analysis of flux (speed * density) of marine organism, there are more marine organisms during ebbs than those in falls.

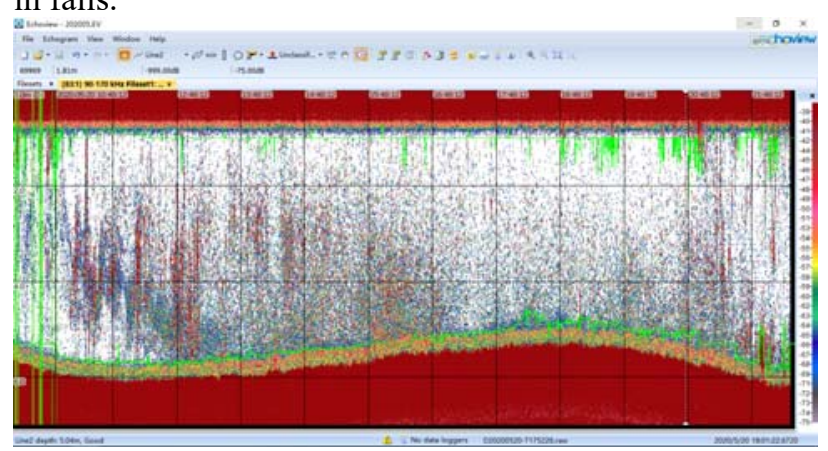

Fig.2 Compressed echo of monitoring results

The echo mappings of small fish clusters in $0-2 \mathrm{~m}$ and 2-4m water depths at about 18:00 are shown in Fig.3-A. The echo mappings of high-density small marine clusters in the 2-6m water depth within a long period from about 22:00 are shown in Fig. 3-B. The relatively strong echo mappings of small fish clusters in the $3-6 \mathrm{~m}$ water depth at 21:00 are shown in Fig. 3-C.

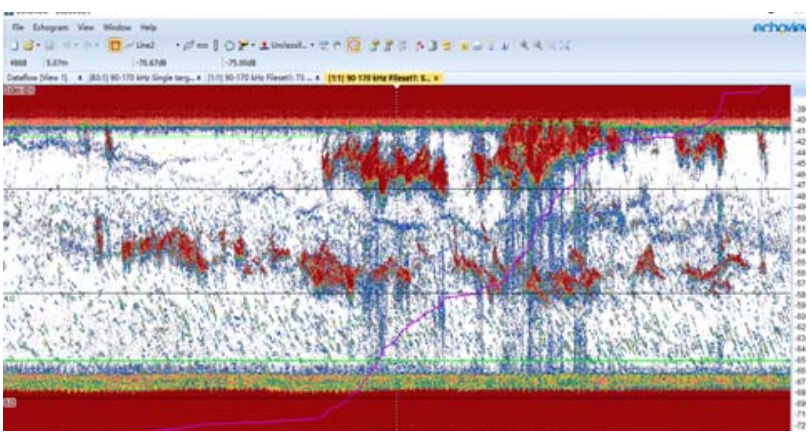

Fig. 3-A The echo mappings of small fish clusters in 0$2 \mathrm{~m}$ water depth at 18:00

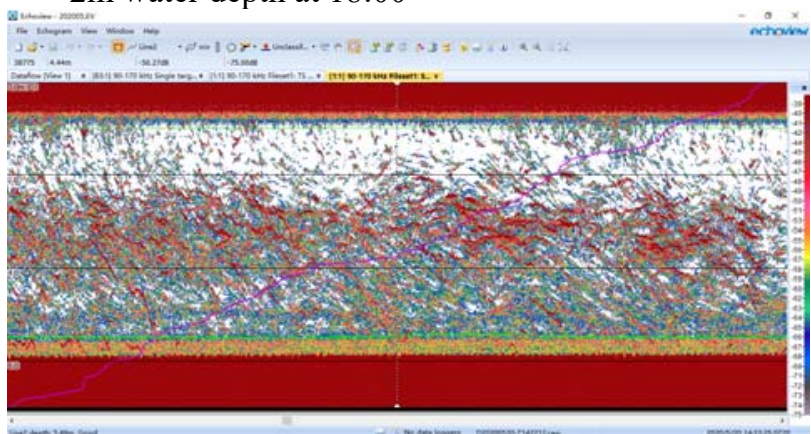

Fig. 3-B The echo mappings of small marine clusters in the 2-4m water depth at 22:00

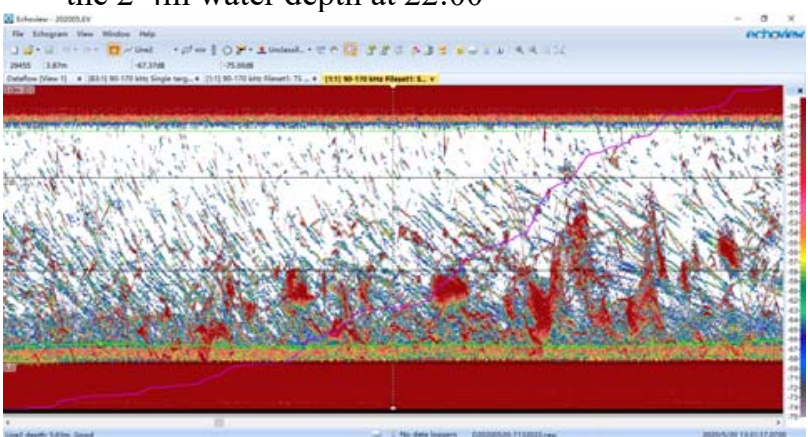

Fig. 3-C The echo mappings of small marine clusters in the 4-6m water depth at 21:00

It can be seen from Fig. 3 that marine organisms in the open seawater channel mainly exist in small clusters. Small fishes and cuttlefish have strong photokinesis, and they will form relatively strong gathering effect at night under lights of boat in the channel ${ }^{8}$.

\subsection{Calculation methods of marine organism density}

In the data post-processing software Echoview, the whole data depth was divided into three levels at an interval of $2 \mathrm{~m}$, which are upper layer, middle layer and lower level ${ }^{9} 10$. Next, marine organism density in each time-space area was calculated every hour. The calculation formulas are shown as follows:

$$
\begin{gathered}
\rho=\frac{\mathrm{sV}}{\sigma_{\mathrm{bs}}} \\
s v=10^{<s v>/ 10} \\
\sigma_{b s}=10^{<T S>/ 10}
\end{gathered}
$$


where sv is the reverse volume scattering intensity of fish clusters. $\sigma$ bs is the reverse scattering section and its numerical value is equal to the linear value of target intensity.

\subsection{Reverse volume scattering intensity of marine organisms}

Changes of mean reverse volume scattering intensity of marine organism (SV) that pass through beam every hour are shown in Fig. $4^{17}$. According to periodic changes of tides, SV declines gradually during falls and increases during ebbs, indicating that small fishes (larva) rush into the open water channel with tides during ebbs. The maximum $\mathrm{SV}$ is about $-43 \mathrm{~dB}$, which is at the sunset and sunrise periods. This is closely related with clustering of zooplankter. Compared to ordinary clean marine water, SV generally is nearly 1,000 times higher at about $-75 \mathrm{~dB}$, indicating that the biomass collected from the open channel has reached to a relatively high level.

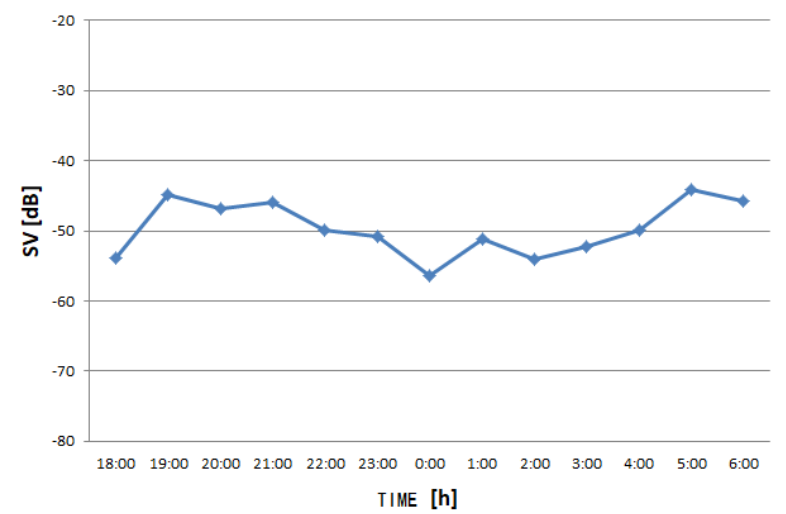

Fig.4 Changes of mean SV at the monitoring point with time

\subsection{Target intensity changes and species distribution of marine organism}

The distribution pattern of hourly single target intensity of marine organisms in the beam from 18:00 to 6:00 of the next morning is shown in Fig.5. Specifically, a relatively high proportion of marine organism is basically kept at lower than $-70 \mathrm{~dB}$, which is mainly contributed by small-sized scattering targets including zooplankter. Deviation of the maximum reflects density differences of different sizes of zooplankter in different periods. However, species of zooplankter and their density proportions are unknown since it doesn't use a zooplankter net for sampling ${ }^{12}$. The target echo between $-70 \mathrm{~dB}$ and $-55 \mathrm{~dB}$ is mainly attributed to small-sized cuttlefish (or larva). Specifically, there are few cuttlefish within 2-3h after 24:00, and there are cuttlefish in other time periods. Due to significant size differences of cuttlefish larva (about $1.5 \mathrm{~cm} \sim 5 \mathrm{~cm}$ ), small zooplankter might overlap with big ones and big zooplankter might overlap with fish larva. During 21:00-22:00 and 1:00$6: 00$, there's a relatively large peak between $-50 \mathrm{~dB}$ and $35 \mathrm{~dB}$, indicating the dense distribution of small fishes (or fish larva) within $3 \mathrm{~cm}-20 \mathrm{~cm}$. In the dense cluster, 5-
$8 \mathrm{~cm}$ small fishes (or larva) take the dominant role. According to capture outcomes by the interceptor behind the sonar, the dense fish cluster is composed of larva of hairtail (about 15-20cm), small carangid and silvery pomfret (about $10 \mathrm{~cm}$ ). The sampling results of the interceptor on the next morning of sampling period are shown in Fig.6. Although there are influences by windstorm and heavy rainfall, basic categories and specification of sampling data can be used (Table 1).

It has to point out that Oratosquilla oratoria (mantis shrimp) is a kind of benthos and they float upward and predate at night. They won't threaten cold-sourced safety under general marine conditions. However, Oratosquilla oratoria will be carried into the intercept to destroy it upon tough marine conditions. Hence, it is necessary to catch and clear Oratosquilla oratoria regularly ${ }^{11}$.

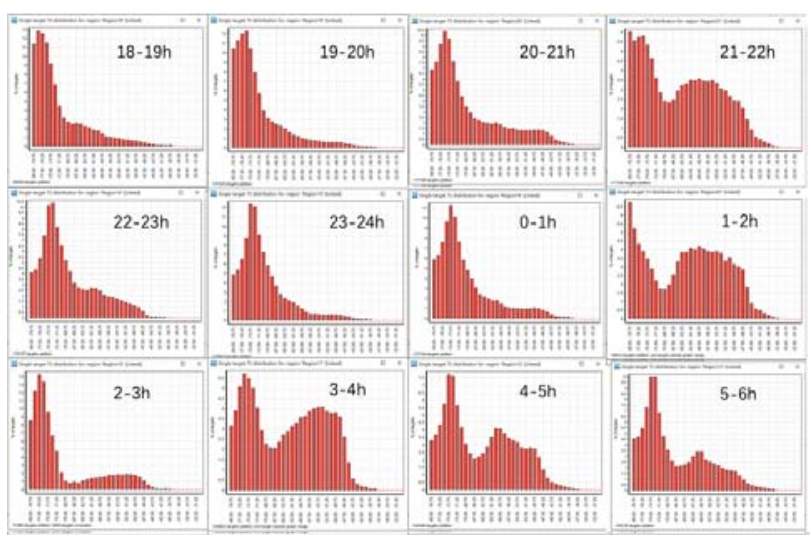

Fig. 5 Changes of target intensity distribution of single marine organism from 18:00 to 6:00 on the next morning

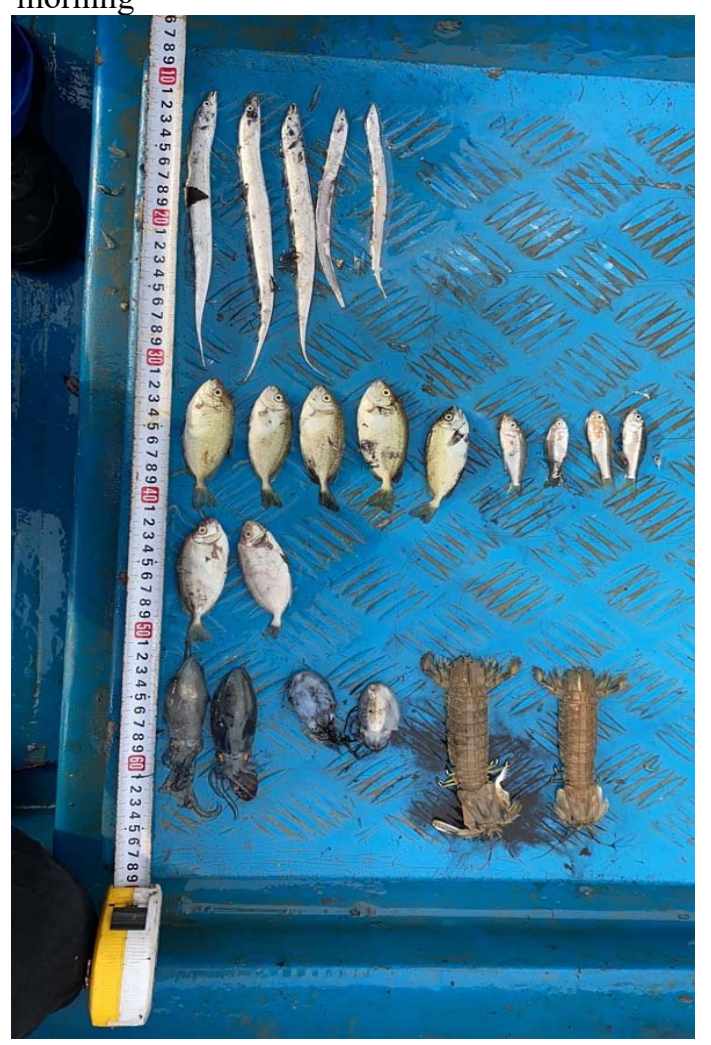

Fig.6 Sampling results from the interceptor after sonar monitoring beam 
Table 1. Sampling results of opening nets intercepted by water intake channels .

\begin{tabular}{|c|c|c|}
\hline Collection & $\begin{array}{l}\text { The first blocking net of } \\
\text { water intake channel }\end{array}$ & Date:2020.5.21 \\
\hline $\begin{array}{c}\text { Serial } \\
\text { number }\end{array}$ & Name & Specification $/ \mathrm{cm}$ \\
\hline 1 & \multirow{5}{*}{ Hairtail } & 21 \\
\hline 2 & & 20 \\
\hline 3 & & 20 \\
\hline 4 & & 14 \\
\hline 5 & & 13.5 \\
\hline 6 & \multirow{5}{*}{ Yellow croaker } & 9 \\
\hline 7 & & 9 \\
\hline 8 & & 9 \\
\hline 9 & & 10.5 \\
\hline 10 & & 11 \\
\hline 11 & \multirow{4}{*}{ Cuttlefish } & 5 \\
\hline 12 & & 6.5 \\
\hline 13 & & 8.3 \\
\hline 14 & & 9.3 \\
\hline 15 & \multirow{2}{*}{ Mantis shrimp } & 13 \\
\hline 16 & & 12.3 \\
\hline 17 & \multirow{2}{*}{ Indian toothless pomfret } & 9.5 \\
\hline 18 & & 9.5 \\
\hline 19 & \multirow{4}{*}{ Chinese male fish } & 6.3 \\
\hline 20 & & 5.6 \\
\hline 21 & & 6.5 \\
\hline 22 & & 6.8 \\
\hline
\end{tabular}

\subsection{Mean density changes of marine organism}

Marine organism densities in different time periods were calculated from Eq.(1) (3). Results are shown in Fig.7. The minimum $\left(0.72 \mathrm{fish} / \mathrm{m}^{3}\right)$ is observed at 19:00, and the maximum (about $0.87 \mathrm{fish} / \mathrm{m}^{3}$ ) is observed during 20:00 to $01: 00$, and the density at 6:00 also is about 0.86 fish $/ \mathrm{m}^{3}$, indicating the relatively high marine organism density. Since size-sized zooplankter in the fish cluster accounts for a relatively high proportion (relatively high proportion of weak scattering targets in Fig.5), it won't threaten the safety of water intaking ${ }^{18}$.

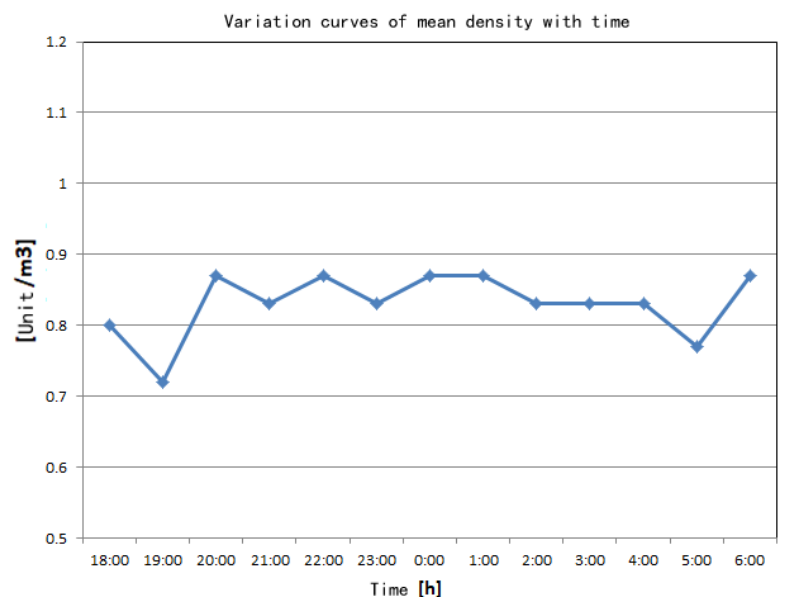

Fig.7 Variation curves of mean density with time in the whole investigation process

\subsection{Density distribution in different water layers}

With considerations to layer setting and setting mode of the interceptor, marine organism density in different water layers was compared and analyzed. Changes of marine density in 0-2m layer, 2-4m layer and 4-6m layer with time were calculated ${ }^{15}$. Results are shown in Fig.8. Generally speaking, densities on surface and bottom layers fluctuate greatly, while density in the middle layer changes slightly. No large density in all three layers simultaneously is observed, indicating that the marine organism cluster is relatively small and no large cluster is formed. Marine organisms belong to normal smallsized clustering behavior, which cannot damage the interceptor and threaten safety of water intake. The maximum density is at 02:00 and the surface density is over $1 \mathrm{fish} / \mathrm{m}^{3}$. This is known as an ultra-dense small fish cluster. However, this fish cluster has no threats to the safety due to the small size (Red echo signal in upper position of Fig.9).

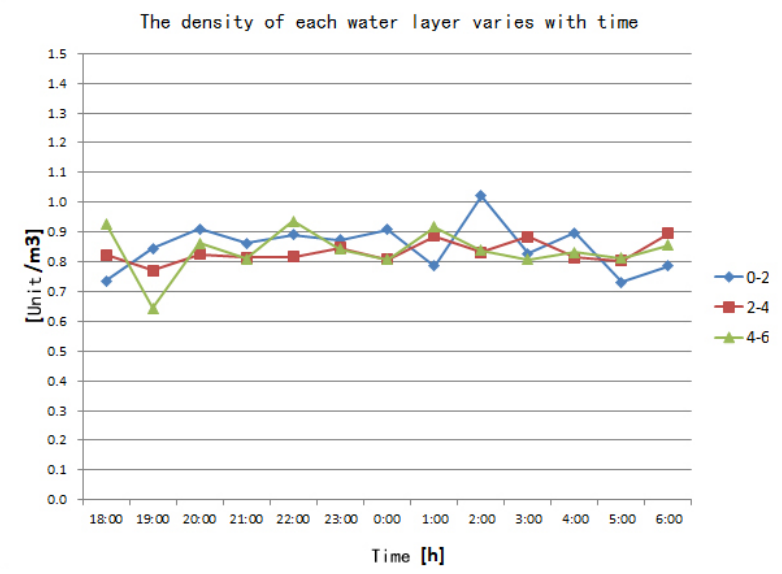

Fig.8 Changes of marine organism densities in different water layers with time

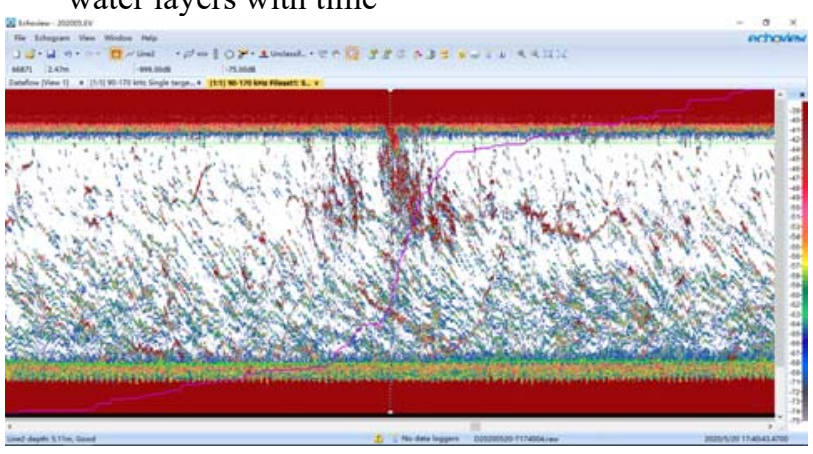

Fig.9 Echo signals of high-density small-sized small fish cluster at 02:00 in the 0-2m layer

\section{Conclusions}

During this investigation, there's a heavy rain and strong storms in the second half of the night, which caused great losses to marine organism samples in the interceptor. In particular, the Oratosquilla oratoria larva is relatively weak and they might suffer great losses by 
tide in the fish bags of the interceptor. Therefore, it cannot reflect organism density in the echo mapping fully ${ }^{13}$. Lights on boat during night might induce great clustering effect to zooplankter and small fishes, thus resulting in high density of measured fish clusters, basically keeping at $0.83 \mathrm{fish} / \mathrm{m}^{3}$ (mean) ${ }^{11}$. Based on echo type, density calculation and sampling results from interceptor, many small fishes (larva) and many larva of other marine organism (e.g. cuttlefish) are collected from the open water channel. Moreover, there's a great deal of Oratosquilla oratoria that has to be cleaned regularly ${ }^{16}$. This can prevent formation of a specific small-sized sheltering fishing farm in the channel ${ }^{19}$, decrease cleaning pressure of interceptor, and increase safety guarantee level to the channel.

\section{References}

1. Li Bin, Chen Guobao, Guo Yu, Chen Zuozhi, Zhang Jun, Wang Dongxu. Hydroacoustic Assessment on Spatial and Temporal Distribution and Resource Reserves of Fishery Resources in the Middle Area of the South China Sea $[\mathrm{J}]$. Southern Fisheries Science, 2016(04)

2. Zhang Jun. Acoustic Assessment on Anchovy Resources in the Yellow Sea based on Acoustic Data Post-processing System [D]. Shanghai Ocean University,2011.

3. Tan Xichang, Shi Jianquan, Zhang Hong, Tao Jiangping, Yang Jianxin, Qi Hongfang, Li Xinhui. Applications of EY60 Eco-sounding Gear in Fishery Resource Assessment in Qinghai Lake [J]. Journal of Lake Sciences, 2009(06)

4. Liu Shigang, Tang Yong, Chen Guobao, Zhang Jun, Yao Zhuang, Guo Yu, Liu Huabo. Preliminary Study on Vertical Distribution and Diurnal Changes of Acoustic Scattering Layers in Deep Sea Areas of the South China Sea [J]. Advances in Marine Science, 2015(02)

5. Li Yongzhen, Chen Guobao, Zhao Xianyong, Chen Yuzhen, Jin Xianshi. Acoustic Assessment on Small-scaled Non-economic Fishery Resources in Northern Sea Areas of the South China Sea. Journal of Ocean University of China (natural science), 2005(02)

6. Zhang Jun, Qiu Yongsong, Chen Zuozhi, Zhang Peng, Zhang Kui, Fang Jiangtao, Chen Guobao, Cai Yancong, Sun Mingshuai. Progresses in Investigation and Assessment of Marine Fishery Resources in the South China Sea [J]. Southern Fisheries Science, 2018(06).

7. Zhang Jun, Chen Bumao, Chen Guobao, Yu Jie, Fan Jiangtao, Qiu Yongsong. Background Noises Deduction Method based on Echoview Acoustic Data Post-processing System [J]. Advances in Fishery Science, 2014(01).

8. Chen Guobao, Li Yongzhen, Zhao Xianyong, Chen Yuzhen, Jin Xianshi. Acoustic Assessment on Five Categories of Important Economic Fishery
Resources in the South China Sea [J]. Acta Oceanologica Sinica (in Chinese), 2006(02).

9. Zhang Peng, Yang Lin, Zhang Xufeng, Tan Yongguang. Development Status and Prospects of Tuna and Symlectoteuthis oualaniensis Resources in South China Sea $[\mathrm{J}]$. South China Fisheries Science, 2010(01)

10. Zhang Jun, Chen Zuozhi, Chen Guobao, Qiu Yongsong, Liu Shigang, Yao Zhuang. Research on Relevant Acoustic Measurement and Assessment Technologies for Symlectoteuthis oualaniensis in the South China Sea [J]. Southern Fisheries Science, 2014(06).

11. Mesopelagic fish biomass in the southern California current ecosystem $[\mathrm{J}]$. Peter Davison,Ana LaraLopez,J. Anthony Koslow. Deep-Sea Research Part II . 2015

12. What information do artificial reef managers really want from fishery science. STEIMLE F W,,MEIER M H. Fisheries . 1997

13. The role of acoustics in ecosystem-based fishery management. Koslow J A. Ices Journal of Marine Science .2009

14. Analysis of vessel influence on spatial behavior of fish schools using a multi-beam sonar and consequences for biomass estimates by echosounder. Soria M. ICES Journal of Marine Science . 1996

15. Length and species-dependent diurnal variation of catch rates in the Norwegian Barents Sea bottomtrawl surveys. Korsbrekke K,Nakken O.ICES Journal of Marine Science 1999

16. Acoustic distribution of discriminated micronektonic organisms from a bi-frequency processing: The case study of eastern Kerguelen oceanic waters $[\mathrm{J}]$. Nolwenn Béhagle,Cédric Cotté,Anne Lebourges-Dhaussy,Gildas Roudaut,Guy Duhamel,Patrice Brehmer,Erwan Josse, Yves Cherel. Progress in Oceanography . 2017

17. In situ acoustic target strength measurements of tuna associated with a fish aggregating device. E. Josse,A. Bertrand. ICES Journal of Marine Science . 2000

18. Acoustical measurement of fish abundance. Maclennan D N. The Journal of The Acoustical Society of America . 1990

19. Fisheries acoustics:theory and practice. Simmonds J,Maclennan D. . 2005

20. Simrad ER60 scientific echo sounder software reference manua. Simrad. 2008 